\title{
SOBRE LA FILOSOFÍA DE DONALD DAVIDSON
}

OLBETH HANSBERG

Instituto de Investigaciones

Filos 6ficas, UNAM

La mayoría de los artículos que se incluyen en Actions and Events y Truth and Interpretation* fueron presentados en un congreso sobre la filosofía de Donald Davidson que tuvo lugar en la Universidad de Rutgers, New Jersey, en abril de 1985. Las dos antologías se dividen en secciones que corresponden a las secciones de los dos libros de Davidson Essays on Actions and Events, Oxford, 1980 e Inquiries into Truth and Interpretation, Oxford, 1984, y el propósito de los editores es que han de utilizarse como libros complementarios a estos. Así, el primer volumen, Actions and Events, se divide en tres partes: Intención y acción, Suceso y causa y Filosofía de la psicología. Cada una incluye una útil introducción de los editores en la que resumen las ideas de Davidson en el contexto de la discusión actual de los temas a los que se refiere cada una de las partes. Este libro incluye, además, dos artículos más recientes del propio Davidson ('Deception and Division'** y 'Rational Animals') y su réplica al artículo de Quine sobre la identidad de los sucesos.

El segundo volumen, Truth and Interpretation, se divide en cinco secciones: Verdad y significado, Aplicaciones, Interpretación radical, Lenguaje y realidad y Los límites de lo literal. En una sola introducción al volumen en su conjunto, LePore presenta las ideas de Davidson con relación a los te-

- Actions and Events. Perspectives on the Philosophy of Donald Davidson, edited by Ernest LePore \& Brian McLaughlin, Blackwell, 1985; Truth and Interpretation. Perspectives on the Philosophy of Donald Davidson, edited by Ernest LePore, Blackwell, 1986.

* Los artículos señalados con (*) los presentó Davidson en México y las traducciones al español se publicarán en los libros correspondientes al Primero y Quinto Simposio Internacional de Filosofía del IIF. 
mas de las distintas partes. También en este libro se incluyen tres artículos de Davidson posteriores a Inquiries into Truth and Interpretation ("A Coherence Theory of Truth and Knowledge'**, 'Empirical Content' y 'A Nice Derangement of Epitaphs').

Dado que en estos dos libros se publican 58 artículos - sin contar las 4 introducciones - que abarcan toda la amplísima temática de Davidson, es imposible reseñarlos todos en una nota. Me ocuparé, por tanto, sólo de algunos de los temas. Sin embargo, debido a que la filosofía de Davidson es sumamente coherente y a que todos los temas están íntimamente relacionados, hay siempre algo de arbitrario e incompleto en la presentación de algún tema aislado. Por otro lado, al escoger ciertos temas y problemas estoy eligiendo sólo algunos artículos y dejando fuera otros que pudieran ser igual o aún más importantes.

Uno de los temas de la filosofía de Davidson que ha tenido una enorme influencia es el de la explicación de la acción. ${ }^{1}$ Las acciones son sucesos que pueden describirse de múltiples maneras y que se distinguen porque satisfacen al menos una descripción relativa a la cual la acción es inten. cional. Ahora bien, cuando hacemos algo intencionalmente lo hacemos por una razón. Entendemos las acciones intencionales cuando entendemos el propósito que tenía el agente al actuar, esto es, cuando entendemos las razones que tenía para actuar de esa manera. Una razón es una combinación de creencias y deseos o pro-actitudes. Este último es un término técnico que Davidson introduce ya que una proactitud puede incluir no sólo un deseo sino también un impulso, deber, obligación o cualquier otra actitud a favor de una acción. Mencionar razones para explicar una acción implica, entonces, hacer referencia a los deseos y creencias que llevaron a una persona a actuar de cierta manera. Un ejemplo muy simple de este tipo de explicación es decir que Juan bebió la cerveza que tenía enfrente porque deseaba

\footnotetext{
${ }^{1}$ Essays on Actions and Events.
} 
apagar su sed y creía que beber la cerveza era la manera de hacerlo. Ahora bien, este deseo y creencia que explican la acción de Juan, están a su vez relacionados con otros deseos y creencias. Así, Juan cree que beber la cerveza apagará su sed, sólo si cree que lo que tiene enfrente es una cerveza, si cree que la cerveza es bebible, que está en buen estado, que la cerveza quita la sed, etc., etc. En cualquier explicación de la acción - por más simple que ésta sea - nos encontramos con que cada uno de los deseos y creencias que le adscribimos a un agente para construir una razón, está conectado, por su contenido proposicional, con un número indefinido de otros deseos y creencias. Y cada uno de los deseos y creencias que explican una acción puede, a su vez, explicarse mediante combinaciones de creencias, deseos y otras actitudes proposicionales que constituyen razones para ellas. Así, cuando Juan dice 'El agua está fría', la razón de que lo diga es que desea decir que el agua está fría y cree que proferir estas palabras es una manera de hacerlo. Pero quiere decir que el agua está fría porque desea advertir a María. Y desea advertirle porque cree que podría pescar un resfriado, etc.

Ahora bien, muchas veces el mencionar el deseo inmediato a la acción -en el caso anterior, el deseo de decir que el agua está fría - nos proporciona poca o ninguna información acerca del porqué de la acción. Mencionamos entonces otros deseos y creencias de los cuales se deriva este deseo. Por ejemplo, el deseo de advertir a María. Esto sí es informativo dado que puede haber una infinidad de otros deseos y creencias que pueden haber actuado en combinación para producir el deseo que se ve en la acción. Podemos siempre proporcionar más razones para los deseos y creencias que aparecen en la explicación y, como cada uno de ellos implica a su vez otros que le dan contenido, podemos entender que cada acción ocupa un lugar en una red de razones que pueden recorrerse de múltiples maneras. Sin embargo, Davidson sostiene que para que una razón explique una acción no basta con que exista una razón y ocurra la acción, sino que lo importante es que la acción se haga por esa razón. Esto es, un agente puede tener una serie de deseos y creen- 
cias que hacen razonable una acción y, sin embargo, no actuar, o puede actuar pero no por esa razón. Entonces, no basta con que el agente tenga actitudes proposicionales adecuadas y que ocurra la acción, sino que el agente debe actuar por esa razón. Ahora bien, Davidson piensa que la manera de explicar ese 'por' es decir que los deseos y creencias causan la acción. Esto es, las razones causan acciones, y la racionalización es una especie de explicación causal.

Para que esto sea plausible hay que aclarar algunos puntos:

En primer lugar el uso del concepto de causalidad produce un contexto extensional. Esto es, la causalidad es una relación entre sucesos particulares, irrepetibles y fechados. Puedo decir que si un suceso $a$ causó un suceso $b$ esto es verdadero cualesquiera que sean las maneras en que describa los sucesos. El concepto de explicación, en cambio, crea un contexto intensional. La explicación causal relaciona oraciones y, por tanto, las descripciones de los sucesos son relevantes a la verdad de un enunciado explicativo. Siempre que explicamos un suceso lo explicamos bajo cierta descripción que tiene que ver con lo que queramos explicar. ${ }^{2}$ Así, cuando lo que queremos explicar es una acción, usaremos descripciones que describan las causas en términos de actitudes proposicionales y a la acción como una acción intencional.

Davidson sostiene que los enunciados causales singulares requieren de leyes, pero le da una lectura especial a este requisito: 'e...'A causó B' implica que existe una ley causal ejemplificada por alguna descripción verdadera de A y B.",3 Esta lectura es la que se adecua a la mayoría de las explicaciones causales, entre ellas a las racionalizaciones. Pero

\footnotetext{
${ }^{2}$ Hay toda una sección dedicada al tema de los sucesos y al de la causalidad. Aquí sólo quiero señalar el artículo de Quine, pp. 162-171, quien defiende la tesis de que dos sucesos son idénticos si y sólo si ocurren en la misma región espacio-temporal. Y también la réplica de Davidson, pp. 172-176, quien acepta esta definición de identidad pero, al mismo tiempo, defiende sus razones por las que los sucesos han de considerarse como una categoría ontológica básica distinta a la de los objetos.

3 'Actions, Reasons and Causes', en Essays on Actions and Events, p. 16.
} 
"las leyes cuya existencia se requiere si las razones han de ser causas de las acciones, ciertamente no manejan los mismos conceptos que las racionalizaciones". Entonces, una cosa es decir que un suceso causó otro suceso y otra cosa muy distinta es decir que hemos caracterizado a los sucesos de una manera adecuada para ejemplificar una ley causal.

Para Davidson, las leyes causales estrictas sólo pueden ser leyes físicas porque no puede haber conexiones nomológicas entre lo mental y lo físico. Este es el principio de la anomalía de lo mental que, en síntesis, afirma que las explicaciones de las actitudes proposicionales están gobernadas por principios constitutivos de la racionalidad que son holistas, interpretativos y esencialmente normativos y que no pueden expresarse en leyes estrictas. Estas características de lo mental no tienen un correlato en el mundo físico. "No puede haber conexiones fuertes entre los dos dominios si cada uno quiere mantenerse fiel al tipo de evidencia que le es propia."

En su artículo, 'Davidson on Intentional Behaviour', Stoutland acepta la versión de Davidson acerca de la racionalización de las acciones, pero rechaza la idea de que esta versión sea compatible con una teoría causal de la acción. Sostiene que la tesis de que el agente actúa por una razón sólo si la razón causa la acción es una tesis inútil que sólo causa problemas. Su argumento es el siguiente: 1) El que la acción sea causada por una razón es lo que distingue a una acción intencional de otra que no lo es. 2) Que, debido a la anomalía de lo mental, no hay leyes psicofísicas y, por tanto, todas las leyes son físicas. 3 ) Si una razón causa una acción entonces debe haber descripciones físicas tanto de las actitudes proposicionales como de la acción que las subsuman bajo una ley física. De 1,2 y 3 debemos concluir, según Stoutland, que la distinción entre una conducta intencional y otra que no lo es, es la distinción entre una conduc-

\footnotetext{
4 Ibid., p. 17.

5 'Mental Events', op. cit., p 98.

${ }^{6}$ Actions and Events, pp. 44-59.
} 
ta a la que se aplica una ley física específica y una conducta a la que no se le aplica esa ley. Cuando distinguimos entre conducta intencional bajo una descripción y conducta no intencional debemos tomar en cuenta dos cosas: "la primera es la evidencia que tenemos para aplicar la distinción en un caso particular para determinar efectivamente si la conducta es intencional o si el agente actuó por una razón. La segunda son las condiciones de verdad de la distinción - las condiciones necesarias para que la conducta de un agente sea intencional o para que una razón sea su razón para actuar. El análisis causal de Davidson debería requerir de una apelación a leyes físicas en los dos casos. Nuestra evidencia de que la conducta de un agente es intencional debería incluir una referencia a leyes físicas. $Y$ una condición necesaria para que la conducta sea intencional, debería ser la presencia de una ley física apropiada..."7

Me parece que Stoutland se equivoca al plantear el problema de esta manera. Cuando Davidson afirma que la causalidad implica leyes, está aceptando una concepción humeana de la causalidad. Pero independientemente del tipo de análisis que acepte, la causalidad es para él una relación entre sucesos, y el 'causó' que aparece en el enunciado causal particular 'A causó B' es un término primitivo. Cuando lo anterior se aplica a la relación entre acciones y razones, podemos aceptar un enunciado causal particular que dice que una razón causó una acción, sin necesidad de hacer ninguna referencia a leyes. Podemos no conocer ni las descripciones físicas adecuadas de los sucesos que tienen la relación causal, ni las leyes relevantes. Lo único que sostiene Davidson - dado su análisis nomológico de la causalidad - es que si una razón causa una acción, entonces habrá al menos una descripción de esos sucesos que será adecuada para subsumirlos bajo leyes físicas relevantes. Pero de esto no se sigue que debamos atribuirle un rol epistemológico a la teoría causal de la acción. Las leyes no son ni pueden ser parte de nuestra evidencia para que podamos

\footnotetext{
${ }^{7}$ Ibid., p. 48.
} 
decir que ciertas actitudes proposicionales causaron una acción. Davidson, como el mismo Stoutland reconoce, nos ha proporcionado múltiples argumentos en contra de esta idea. ${ }^{8}$

Por otro lado, las leyes tampoco juegan un papel cuando damos las condiciones de verdad de un enunciado causal particular. Y aquí hay que enfatizar la palabra 'condiciones'. Las condiciones de verdad se dan en forma austera mediante una oración-T. 'A causó B' es verdadera si y sólo si A causó $B$. 0 , si insistimos en que dar las condiciones de verdad hace transparente la forma lógica de la oración, tendremos, por ejemplo, que 'A causó $B$ ' es verdadera si y sólo si $($, e) Ae causó (,e') Be'. Lo que me importa señalar es que esta versión mantiene también el 'causó' primitivo.

Ahora bien, si lo que Stoutland quiere decir con condiciones de verdad es otra cosa, por ejemplo, cómo distinguimos cuándo una oración efectivamente es verdadera, entonces hemos regresado a un plano epistemológico en el que no queda claro cuál debería ser el papel de las leyes física. Parece, sin embargo, un hecho indudable que nosotros constantemente aceptamos como verdaderos enunciados causales particulares sin conocer la historia nómica que los apoya.

Pero, si Stoutland acepta la tesis de la racionalización de las acciones pero no la teoría causal, entonces tendrá el problema de explicar la distinción entre una acción que se hace por una razón y una que no se hace por esa razón. Él afirma que hay una 'diferencia significativa' entre los dos casos pero no da una versión clara de cómo ha de explicarse. ${ }^{9}$ Nos propone un intento de explicación argumentando contra la tesis de Davidson que afirma que las descripciones intencionales de una acción describen a la acción mencionando las causas o efectos de la acción. Sostiene que hay

\footnotetext{
${ }^{8}$ Por ejemplo, los relacionados con la naturaleza dispar de lo mental y lo físico; la caracterización de la manera especial en que adscribimos y explicamos actitudes proposicionales, etc.

${ }^{9}$ Otro problema que Stoutland deberá solucionar es el de distinguir cuál fue la razón que produjo una acción, en el caso en que tenemos varias razones para hacer algo pero lo hacemos por una.
} 
descripciones intencionales de las acciones que no implican una referencia a causas o efectos. Así, cuando describimos una acción como 'pegándole intencionalmente a alguien', no estamos comprometidos con ninguna tesis particular acerca de qué causó su conducta. ". . ni la evidencia ni las condiciones de verdad para las descripciones psicológicas requieren, en general, que la conducta tenga alguna causa en particular." ${ }^{10}$ Aparte de lo confusa que me parece esta tesis, entre otras cosas porque 'pegándole intencionalmente a alguien' no es una descripción de una acción particular si la acción no fue hecha por una razón determinada, ¿cómo explicar este 'por' si no hubo una causa particular de la conducta?

En relación con las descripciones que mencionan efectos, Stoutland dice que aunque 'hacerle daño a alguien' no describe una acción a menos que haya un daño como efecto, las descripciones que contienen la expresión 'tratar de' como parte de la descripción de una acción, por ejemplo, 'trató de golpear a Juan', no describen la acción en términos de sus efectos, ya que cuando Pedro trata pero no logra pegarle a Juan, ésta sigue siendo, sin embargo, la descripción intrínseca de la acción. Quizá las descripciones con 'tratar de' sean descripciones intrínsecas de las acciones, pero me parece que a Stoutland le quedará la tarea de explicar la diferencia entre tratar de pegar con éxito y tratar de pegar y fallar, sin mencionar que en un caso la acción tuvo el efecto esperado y en el otro no."

Una teoría de Davidson que ha sido ampliamente discutida es la que se refiere a las relaciones entre lo mental y lo físico: el 'monismo anómalo'. Se trata de una teoría fisica-

\footnotetext{
10 Ibid. p. 58.

11 Hay dos artículos más que discuten el tema de las intenciones en Davidson. George Wilson pretende también que el examen de la naturaleza y función del concepto de intención of rezca una alternativa a la teoría causal. M. Bratman, por otro lado, of rece una explicación de lo que es hacer algo intencionalmente; de lo que es tener la intención de hacer algo en el futuro; y de cómo se relacionan estos dos fenómenos. El artículo de Bratman es casi el mismo que apareció en Essays on Davidson: Actions and Events, Vermazen y Hintikka (eds.), Oxford, 1985. La diferencia es que le agrega una última parte en la que trata de contestar a las objeciones de Davidson.
} 
lista que defiende identidades particulares entre sucesos mentales y físicos. El argumento en que se apoya esta teoría parte de tres principios que parecen inconsistentes pero que Davidson acepta como verdaderos. Son: 1) el principio de interacción causal entre sucesos mentales y sucesos físicos; 2) el principio de la anomalía de lo mental, y 3) el principio del carácter nomológico de la causalidad. Con base en estos principios argumenta que: si un suceso mental $m$ causa un suceso físico $f$ (principio 1) y, como no existen las leyes psicofísicas (principio 2), no puede haber una ley psicofísica que apoye esta relación causal particular, entonces - dado el principio del carácter nomológico de la causalidad (principio 3) - la ley que la apoya deberá ser física y, por tanto, el suceso mental que cae bajo una ley física deberá también ser un suceso físico.

Hay varios artículos que discuten este argumento. Kim, por ejemplo, analiza una vez más la posibilidad de leyes psicofísicas. ${ }^{12}$ Johnston hace una comparación entre el materialismo australiano que proponía identidades de tipos y el monismo anómalo que propone identidades particulares, y discute los problemas que debe solucionar una teoría fisicalista para dar cuenta de las características propias de lo mental. ${ }^{13}$

Davidson sostiene que la tipificación de los sucesos es dis. tinta según la terminología que emplean las distintas ciencias y el sentido común para describir los sucesos. Pero todos los sucesos - a pesar de las tipificaciones distintascaen, sin embargo, bajo el dominio de la física. Hornsby discute algunas dificultades con esta tesis que ponen en cuestión el principio del carácter nomológico de la causalidad. ${ }^{14}$

Pero el artículo que me parece más interesante de esta sección es el de McDowell, 'Functionalism and Anomalous Monism', ${ }^{15}$ en el que defiende la tesis de la anomalía de lo

\footnotetext{
12 'Psychophysical Laws', en Actions and Events, pp. 369-386.

13 'Why Having a Mind Matters', ibid., pp. 408-426.

14 'Physicalism, Events and Part-Whole Relations', ibid., pp. 444-458.

${ }^{15}$ Ibid., pp. 387-398.
} 
mental frente a la posibilidad de un reduccionismo funcionalista que propone Brian Loar en su libro Mind and Meaning. La tesis de Loar es que la posibilidad de una interpretación funcional de las actitudes proposicionales permite un tipo de reducción fisicalista. McDowell muestra cómo una interpretación funcional de las actitudes proposicionales es imposible si tomamos en serio el ideal constitutivo de la racionalidad en la formación de nuestro pensamiento acerca de las actitudes proposicionales. Reconocer el status ideal del concepto constitutivo de la racionalidad es apreciar el hecho de que los conceptos de actitudes proposicionales tienen un ámbito propio en un tipo especial de explicaciones, a saber, las explicaciones en las que las cosas se hacen inteligibles al revelarse -0 al aproximarse a ser-como racionalmente debieran ser. Ya he dicho algo sobre este punto y volveré a él más adelante. Ahora sólo me referiré a las dudas que expresa McDowell acerca del carácter nomológico de la causalidad y acerca de la tesis monista de Davidson.

Como ya dije, McDowell defiende la tesis de la anomalía de lo mental al enfatizar cómo las explicaciones de actitudes proposicionales están gobernadas por el ideal constitutivo de la racionalidad que no tiene eco en la teoría física. Sostiene, sin embargo, que esto no va en contra de la tesis de que este tipo de explicaciones funcionan citando causas. Piensa que sí es explicativo mencionar causas aun cuando no aceptemos una concepción humeana de la causalidad, esto es, una concepción que se apoye en la idea de que ". . .es en virtud de ser una instancia de una generalización acerca de cómo tienden a suceder las cosas, (...) que la relación entre un determinado par de sucesos es una relación causal" ${ }^{16}$ Afirma que esta idea de causalidad, incorporada en el principio de Davidson, es un prejuicio - el cuarto dogma del empirismo- que podríamos abandonar reteniendo la idea de que las relaciones causales se dan en la experiencia. El principio se basa en la tesis de Hume de que - dado

${ }^{16}$ Ibid., p. 394. 
que las relaciones causales no se dan en la experiencia- la causalidad no consiste más que en un tipo adecuado de generalidad, y esta tesis "parece inextricablemente ligada a un 'dualismo de esquema y contenido, de un sistema organizador y algo que espera a ser organizado' que Davidson nos ha mostrado que es insostenible. Sin ese dualismo, no hay ningún atractivo obvio en la idea de que las relaciones causales no se dan en la experiencia". ${ }^{17}$

Ahora bien, al poner en duda el principio de Davidson, McDowell rechaza también el monismo que se apoya en él. Dada nuestra concepción científica y del sentido común acerca del mundo, el atractivo de una tesis fisicalista consiste en que no tiene sentido para nosotros la idea de una subs. tancia no-física. Para evitar una tesis no-naturalista como la que proponía el dualismo cartesiano, suponemos que debe ser correcta alguna tesis fisicalista. Pero si analizamos más a fondo la imagen cartesiana, esta recomendación a favor del fisicalismo desaparece. Lo que está realmente en cuestión, según McDowell, es la influencia que tiene la idea "de que la realidad es objetiva, en el sentido de ser completamente descriptible desde ningún punto de vista en particular. Esta idea está en tensión con la intuición natural de que lo mental es tanto real como esencialmente subjetivo". ${ }^{18} \mathrm{El}$ dualismo cartesiano resulta del intento de resolver esta tensión. La idea del acceso privilegiado supuestamente da cuenta de la subjetividad de lo mental, mientras que el objeto de ese acceso se concibe como algo que está ahí independientemente, esto es, como una realidad objetiva. Como no es plausible la idea de un acceso privilegiado a algo físico, surge la noción de una substancia no-física.

Recurrir al fisicalismo sólo evita el defecto de postular una substancia no-física, pero no lo que McDowell considera como el defecto principal de la imagen cartesiana de lo mental, a saber, el tratar de forzar a lo mental en un molde objetivo.

17 Ibid., p. 398.
${ }^{18}$ Ibid., p. 395. 
La tesis de la irreductibilidad de lo mental de Davidson respeta la intuición de la subjetividad de lo mental, ya que para entender a los otros tenemos que tomar en cuenta el contenido específico del punto de vista de un sujeto sobre el mundo, y estar abiertos a la posibilidad de aprender de él y modificar nuestras propias actitudes. El tipo de comprensión y explicación "para el cual la racionalidad juega un papel constitutivo, requiere de una sensibilidad al detalle específico de la postura subjetiva de los otros (...) que se falsificaría si uno supone que ...(estas) ... explicaciones funcionan colocando sus explananda en una estructura especificable desde contenidos externos". ${ }^{19}$

Así, si sostenemos que las ciencias físicas pueden, en principio, explicar la totalidad de la realidad objetiva, y si aceptamos la idea de que los estados mentales "son irreductiblemente mentales en virtud de una subjetividad ineliminable" y no pretendemos, por tanto, que lo mental pueda representarse como un aspecto de la realidad objetiva, entonces podemos concederle a lo mental una "realidad completa y autosuficiente" y concebirlo "como un aspecto diferente del mundo natural". ${ }^{20}$

Otro tema al que me referiré en este trabajo es el de la interpretación radical. Davidson propone un modelo de la manera en que procedemos cuando queremos comprender la conducta lingüística de los otros. Describe un caso extremo, el de la interpretación radical, que es la situación en la que un intérprete se enfrenta por primera vez con una comunidad de hablantes cuyo lenguaje no conoce y desea interpretar. Este caso le sirve para mostrar con mayor claridad algo que es una situación usual, a saber, la manera en que procedemos siempre que queremos interpretar a los otros aun cuando hablen el mismo lenguaje.

La idea es que existe una interdependencia entre dos aspectos de la interpretación de la conducta lingüística: la atribución de actitudes proposicionales y la interpretación

19 Ibid., p. 396.

${ }^{20}$ Ibid., p. 396. 
del habla. Lo que una persona dice no puede interpretarse sin tomar en cuenta lo que esa persona cree, desea, intenta, etc., $\mathrm{y}$ las distinciones finas entre creencias son imposibles si no entendemos lo que dice. En la situación de interpretación radical, esto es, en el caso en que no podemos asumir como dados ni significados ni creencias, ¿cómo podemos interpretar las palabras de alguien o atribuirle creencias y otras actitudes proposicionales de una manera inteligible? Necesitamos una teoría que simultáneamente explique las actitudes e interprete el habla, una teoría que se base en una evidencia que no asuma ninguna de las dos.

La noción mentalista de considerar como verdadera una oración no interpretada, es la que le sirve a Davidson para generar los datos para un esquema de interpretación..$^{21}$ Esta actitud es una creencia, pero una creencia muy general que el sujeto tiene frente a todas las oraciones que considera verdaderas; una actitud que el intérprete puede distinguir antes de saber de qué verdad se trata.

Ahora bien, la tarea del intérprete consiste, en primer lugar, en darse cuenta de cuáles rasgos de la situación del sujeto son los que constituyen una razón para que en un momento dado considere una oración como verdadera. Este método funcionará al principio sólo para oraciones cuyo valor de verdad cambia en situaciones distintas, por ejemplo, oraciones como 'Está lloviendo'. De estas conjeturas acerca de qué es lo que causa que un sujeto considere como verdaderas ciertas oraciones, más la tesis de que, si los miembros de una comunidad tienen una relación de este tipo frente a la enunciación de una oración determinada, esto constituye

\footnotetext{
${ }^{21}$ Davidson sostiene que necesitamos una teoría de la interpretación verbal para obtener actitudes y creencias que expliquen nuestras preferencias y elecciones, pero también necesitamos una teoría de grados de creencia para poder usar adecuadamente las relaciones de apoyo evidencial. Dada la interdependencia de nuestras actitudes básicas, no es posible desarrollar cada una independientemente. En 'Towards a Unified Theory of Meaning and Action', Grazer Philosophische Studien, 1979, Davidson propone una teoría según la cual pueden obtenerse grados de creencia en una escala de racionalidad, utilidad en una escala de intervalos e interpretación del habla sin asumir ninguna de estas cosas. Todo lo que tiene el intérprete para empezar es información acerca de qué sucesos en el mundo causan que un sujeto prefiera que sea verdadera una oración más que otra.
} 
prima facie una prueba de que la oración es verdadera, el intérprete obtiene un conjunto de oraciones de la forma: ' $s$ ' es verdadera en L si y sólo si $p$, en las que $s$ se sustituye por el nombre de una oración del lenguaje que hay que estudiar y $p$ por una oración del lenguaje del intérprete que concierne a los rasgos salientes de la situación del sujeto. Por ejemplo: '_ es verdadera para A en $t$ si y sólo si está lloviendo cerca de A en $t$.

Sin embargo, para obtener este conjunto de enunciados, el intérprete deberá hacer ya una serie de suposiciones acerca de cómo piensa él que el sujeto percibe la situación. Esto es, cuando queremos interpretar la conducta verbal de una persona y hacemos una conjetura acerca del significado de sus palabras, estamos haciendo también una suposición acerca de sus creencias y deseos. Lo que hacemos es atribuirle un conjunto de actitudes proposicionales que son, en sus rasgos esenciales, semejantes a las nuestras. Esto es lo que llama Davidson el principio de caridad o de adaptación racional. En la medida en que avanzamos en nuestra interpretación, podemos hacer ajustes y sustituciones en los casos en que podemos hacer más inteligibles los actos y enunciados de una persona si suponemos que difiere de no. sotros en algunas de sus actitudes.

Ahora bien, una teoría que interprete la conducta verbal de un hablante, esto es, una teoría del significado, deberá dar el significado de un número potencialmente infinito de enunciados que pertenecen al repertorio del hablante. Una teoría así tendrá que tomar en cuenta la estructura de las oraciones y las propiedades semánticas de sus partes. Davidson sostiene que debemos descubrir en el lenguaje que hay que interpretar la estructura lógica del cálculo de predicados de primer orden y que la manera de hacerlo es mediante una teoría como la de Tarski pero modificada para aplicarse a lenguajes naturales. Una teoría de este tipo nos permite generar oraciones-T para todas y cada una de las oraciones de un lenguaje. Construimos así una teoría del significado en nuestro lenguaje para su lenguaje y vemos si la teoría nos permite atribuir, de manera inteligible, otras 
actitudes proposicionales y explicar la conducta. Ésta será una manera de probar la teoría. Entonces, si podemos construir una teoría que reconcilie el principio de caridad con las condiciones formales de la teoría, habremos hecho todo para garantizar la comunicación.

Ahora bien, hay una serie de restricciones a la interpretación que limitan nuestras atribuciones de actitudes proposicionales. Mencionaré brevemente algunas:

La interpretación es holista, esto quiere decir que tanto el significado de las palabras como el contenido de las actitudes no pueden considerarse uno a uno, sino sólo en el marco de un conjunto de otros significados o de otras actitudes. Es sólo en el contexto de una teoría completa de las creencias, lenguaje y acciones de una persona, que el intérprete puede decir algo acerca de sus palabras o de los contenidos de sus actitudes.

La interpretación es normativa y las normas que la guían son las normas de la racionalidad. Estas normas guían tanto al intérprete como al interpretado. Al intérprete cuando decide cómo interpretar el pensamiento y acción del agente, pero también guían al agente en sus pensamientos y acciones. Así, para poder interpretar a alguien, tenemos que suponer, por ejemplo, que, en general, tiene creencias razonables y coherentes, que sus preferencias son, en general, transitivas, etc. ${ }^{22}$

Otro principio general es el ya mencionado principio de caridad que aconseja optimizar el acuerdo entre nuestras

\footnotetext{
${ }^{22}$ La teoría de las decisiones y la teoría de la elección racional han tratado de precisar nuestra comprensión de la racionalidad. En varios artículos Davidson utiliza la teoría de las decisiones en la versión de Ramsey y Jeffrey para explicar la conducta racional, pero también examina sus limitaciones.

Jon Elster, en su artículo 'The Nature and Scope of Rational Choice Explanations', $A c$ tions and Events, pp. 60-72, hace una exposición muy clara de la elección racional, de sus limitaciones y del grado de libertad que permite a nuestras interpretaciones.

En su artículo 'Deception and División', op. cit., pp. 138-148, Davidson examina casos de irracionalidad y se refiere a algunos principios de racionalidad que no guían al agente cuando éste actúa de manera irracional, por ejemplo, en los casos de debilidad de la voluntad, auto-engaño y wishful thinking (creencias basadas en deseos). Otros artículos que se ocupan del tema de razones para actuar e irracionalidad son el de Annette C. Baier, pp. 116-129, y la réplica, justa, de David Pears, pp. 130-137.
} 
creencias y las de ellos, ya que el error y las diferencias sólo tienen sentido en un marco de creencias comunes. Este principio no excluye las diferencias de opinión, sino sólo aconseja evitar en lo posible los desacuerdos para los que no hay una explicación racional.

Estos rasgos de la interpretación van en contra de la idea de que pueda haber mentes radicalmente distintas a las nuestras. Michael Root en su artículo 'Davidson and Social Science ${ }^{{ }_{23}}$ considera esta tesis y sus consecuencias para las ciencias sociales, a saber, que no hay culturas cuya visión del mundo pueda ser tan distinta a la nuestra que sea, en principio, imposible entenderlas. Las diferencias de las que hablan tanto autores como Frazer, Turnbull, Levy-Bruhl, etc. en realidad no son tan grandes y suponen un marco común. Esto se muestra en la facilidad con la que describen estas diferencias. Pero, aunque la interpretación requiere que tratemos de entender a los demás haciéndolos semejantes a nosotros, también implica sensibilidad a su punto de vista, aprender de ellos y la posibilidad de modificar nuestras propias mentes. ${ }^{24}$

A pesar de que la teoría de la interpretación es básicamente correcta y ofrece una perspectiva desde la cual puede manejarse la idea de las diferencias individuales y culturales, Root afirma que tiene, sin embargo, la limitación de ser demasiado intelectualista, en el sentido de que las acciones sólo se explican en la medida en que se racionalizan. Existen según él, palabras y actos que tienen una naturaleza ritual o simbólica que se malinterpreta si tratamos de racionalizarlos. Cita una observación de Wittgenstein en contra de las racionalizaciones de prácticas mágicas y religiosas que hace Frazer. Es un error, dice Wittgenstein, "afirmar que el rasgo característico de estas acciones es que surgen de ideas equivocadas acerca de la física de las cosas" ${ }^{25}$ Estos son actos que no se explican por su propósi-

${ }^{23}$ Truth and Interpretation, pp. 272-306.

${ }^{24} \mathrm{Ibid}$., p. 301; pero véase también el artículo de McDowell.

${ }^{25}$ Wittgenstein, Remarks on Frazer's Golden Bough, citado por Root, op. cit., pp. 304. 
to porque no tienen un propósito, sino que su función es la expresión o evocación de un sentimiento. Entenderlos es ver cómo esos actos se asemejan a rituales nuestros que evocan estos sentimientos. Puede haber actos rituales tan distintos a los nuestros, que nos dejan fríos y que, por tanto, no entendemos. Pero esto no quiere decir que las actitudes proposicionales de las personas que hacen este tipo de actos sean inescrutables.

En otra parte de su artículo, Root ofrece una interpretación kantiana de la teoría de Davidson que, según él, muestra la tensión que existe entre la teoría de la interpretación y el realismo de Davidson. Muy resumido, el argumento es el siguiente: Davidson sostiene que el significado de una oración son sus condiciones de verdad. Si una oración tiene condiciones de verdad, es verdadera o falsa. Por otro lado, el realismo afirma que el significado de una oración puede darse a pesar de que ningún hablante de un lenguaje tenga la capacidad de determinar si esa oración es verdadera o falsa. Si aplicamos esta tesis a oraciones acerca de la mente, se sigue que una oración acerca del contenido de la mente puede ser verdadera o falsa aun cuando ningún hablante tenga la capacidad de interpretar esa mente y, como resultado, no tenga la capacidad de descubrir si esa oración es verdadera o falsa. Esto implicaría que la idea de mentes radicalmente distintas tienen sentido aun cuando no tuviésemos la capacidad de saber que son radicalmente distintas. Y ésta es una idea que Davidson rechaza. Por lo tanto, Davidson no puede ser un realista acerca de la mente. ${ }^{26}$ Sería muy largo discutir aquí este argumento dado lo complicado de la cuestión realismo/anti-realismo en relación al lenguaje y lo elusivo que ha sido Davidson con respecto a esta discusión. Sin embargo, es un argumento interesante que - de ser correcto- obligaría a Davidson a elegir entre sostener que, en relación con la mente, si no podemos reconocer el valor de verdad de una adscripción de actitudes proposicionales, no podemos entender el significado

26 Ibid., pp. 296-297. 
de una oración acerca de los contenidos de esa mente. 0 , si es incoherente sostener sólo un anti-realismo acerca de la mente, a abandonar el realismo en general. 0 , por último, a mantener el realismo, pero abriendo entonces la posibilidad de que puede haber esquemas conceptuales intraducibles.

Los artículos de Wallace y Vermazen también retoman el tema de la interpretación. ${ }^{27} \mathrm{~W}$ allace analiza lo que, según él, debería ser la forma correcta de las teorías de Quine y Davidson y cuál debería ser su base evidencial. Hace una comparación entre estas teorías y los esfuerzos de Chadwick por descifrar el Linear B. Wallace piensa que un examen de la evidencia que sirvió para descifrar las tablas, y la manera en que Chadwick encausó esta evidencia para confirmar su teoría, pone a prueba la teoría de la interpretación. Vermazen examina las diferencias entre el caso que presenta Wallace y la teoría de la interpretación y hace una evaluación de las críticas de Wallace. Concluye, en mi opinión correctamente, que no es un caso que ponga en cuestión la teoría. La teoría de la interpretación puede ponerse a prueba en los casos diarios de transacción linguística. Pero es importante tener claridad acerca de cuál es la afirmación empírica que se está haciendo y que debe ponerse a prueba. La teoría de la interpretación es un esquema de teoría que racionaliza cierta práctica, que es la de interpretar el habla de otra persona. "La descripción de la interpretación radical es sólo una manera de presentar la racionalización real, que consiste en enunciar fines para el intérprete, fines tales que sólo la asignación de estados mentales y condiciones de verdad que logra esos fines cuenta como una interpretación. El intérprete debe encontrar enunciados de condiciones de verdad para las oraciones bajo interpretación, y debe saber que éstos son enunciados que resultan de una teoría cuyas consecuencias susceptibles de prueba son correctas... Si el intérprete fracasa en esto, o fracasa en hacer al hablante en gran medida racional o, en general, en ponerlo de acuerdo

${ }^{27} \mathrm{~J}$. Wallace, 'Translation Theories and the Decipherment of Linear B', op. cit., pp. 211 234, y B. Vermazen, 'Testing Theories of Interpretation', op. cit., pp. 235-244. 
con él en relación con los hechos..., no ha logrado interpretar los enunciados del hablante." 28

No me queda más que mencionar algunos de los temas y artículos de los que no me he ocupado. Están, por ejemplo, los artículos más técnicos que examinan temas relativos a las teorías de verdad, forma lógica e inferencia. Pero no quiero dejar de resaltar el excelente artículo de Tyler Burge $^{29}$ y artículos de filósofos como R.E. Grandy, J. Higginbotham y $\mathrm{H}$. Putnam. Tampoco me refiero a la sección sobre los límites de lo literal, que contiene un nuevo artículo de Davidson con comentarios de Dummett y Hacking y un artículo de M. Cavell sobre Freud. En el curso de mi exposición, y en la medida en que me refiero a temas relacionados, menciono otros artículos en notas al pie de página.

Mi opinión general sobre estas dos antologías es que la calidad de los artículos es bastante irregular y que no son muchos aquellos de los cuales puede afirmarse lo que dice Davidson en el prefacio, a saber, que han revelado errores y debilidades en su obra y, en muchos casos, han indicado mejores soluciones que las suyas a problemas que había tratado de resolver. ${ }^{30}$

\footnotetext{
${ }^{28}$ Vermazen, op. cit., p. 243.

29 "On Davidson's "Saying that"', op. cit., pp. 190-210.

${ }^{30}$ Agradezco al Dr. Mark Platts sus comentarios a una primera versión de este trabajo.
} 